\title{
VARIABLES CONDUCTORAS DE LA INDUSTRIA EDITORIAL ESPAÑOLA
}

\section{Driving variables of the Spanish publishing industry}

\author{
Marta Magadán-Díaz y Jesús Rivas-García
}

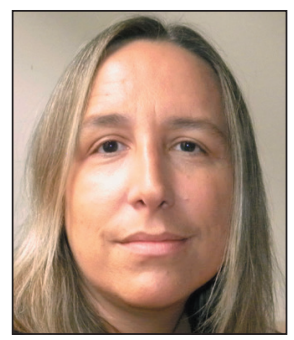

Marta Magadán-Díaz es doctora en Administración de Empresas por la Universidad de Oviedo y profesora de la Universidad Internacional de la Rioja. CEO en la industria editorial, fue presidenta del Gremio de Editores de Asturias y formó parte de la junta directiva de la Federación del Gremio de Editores de España. Fue profesora en la Universidad de Oviedo, y profesora visitante en las universidades de Guadalajara y Colima (México). Desde 2016 dirige Las Tertulias del Campoamor de la Fundación Municipal de Cultura del Ayuntamiento de Oviedo.

https://orcid.org/0000-0003-3178-3215

marta.magadan@unir.net

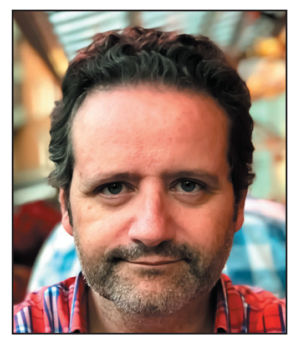

Jesús Rivas-García es doctor en Economía por la Universidad de Oviedo y profesor de la Universidad Internacional de la Rioja. Director del Master Universitario en Dirección de Operaciones y Calidad en la misma universidad, fue profesor en la Universidad de Oviedo y profesor visitante en las universidades de Guadalajara y Colima (México). Desarrolló labores de asesoramiento y consultoría estratégica para empresas e instituciones públicas.

https://orcid.org/0000-0003-0576-5961

jesus.rivas@unir.net

Universidad Internacional de La Rioja Avda. de la Paz, 137. 26006 Logroño, España

\section{Resumen}

Esta investigación tiene por objeto identificar la evolución y tendencias del sector editorial español. Para ello se hará uso en primer lugar de fuentes estadísticas oficiales disponibles sobre el mercado editorial y los hábitos de lectura, de las que: a) se obtendrán los datos necesarios para su tratamiento estadístico y b) se derivará un análisis de situación que permita determinar si el sector editorial privado español se adapta adecuadamente a los cambios del mercado. El trabajo se plantea varias preguntas para el mercado editorial español: a) ¿cómo es la elasticidad del precio de la venta de ejemplares? b) ¿qué impacto tiene el mayor o menor número de editoriales privadas sobre la venta de ejemplares?, c) ¿de qué modo condiciona la venta de ejemplares la evolución de la tirada media? y d) ¿qué impacto tiene el hábito de lectura en las ventas de ejemplares?

\section{Palabras clave}

Editoriales; Libro; Tirada; Hábitos de lectura; España; Análisis de situación; Análisis secundario.

\section{Abstract}

This research aims to identify the evolution and trends of the Spanish publishing sector. For this purpose, firstly, official statistical sources available on the publishing market and reading habits will be used, from which will be obtained: a) the necessary data for their statistical treatment, and b) an answer to whether the Spanish private publishing sector adapts adequately to market changes. The work raises several questions for this market: a) how is the price elasticity of the sale of copies? b) What impact does the greater or lesser number of private publishers have on the sale of copies? c) How does the sale of copies determine the evolution of the average print run?, and d) what impact does the habit of reading have on sales of copies?

\section{Keywords}

Publishing houses; Book; Print run; Reading habits; Spain; Situation analysis; Secondary analysis.

Magadán-Díaz, Marta; Rivas-García, Jesús (2018). "Variables conductoras de la industria editorial española". El profesional de la información, v. 27, n. 6, pp. 1335-1345. 


\section{Introducción}

El sector editorial español tiene un importante peso en las industrias culturales, debido al volumen de negocio que genera, al número de empleos y su contribución al PIB, entre otros factores. La industria editorial española es una de las más potentes de Europa y una de las de mayor proyección internacional, y se sitúa como la cuarta potencia editorial del mundo (tanto en producción como en facturación) por detrás de Estados Unidos, Reino Unido y Alemania. Casi cuarenta editoriales españolas tienen filiales en 32 países, principalmente en Iberoamérica ( $80 \%$ ) y en Europa $(13,3 \%)$. En el ranking de las 56 editoriales más importantes del mundo por volumen de facturación (Milliot, 2017), hay dos grupos españoles, Planeta y Santillana, en los puestos 7 y 24 respectivamente, según los datos correspondientes a 2017.

La actividad editorial en España muestra una serie de tendencias que se están asentando en los últimos años. En especial cabe destacar:

- progresivo acortamiento del ciclo de vida de los libros en el mercado;

- existencia de más títulos, pero también de menos copias -es decir, bajada de la tirada media-;

- descenso de la edición impresa como consecuencia de la creciente apuesta por la edición digital;

- reducción del número de ejemplares vendidos.

El ciclo de vida de los libros se va acortando en los últimos años y las novedades editoriales no superan el mes y medio de estancia en las estanterías de las librerías, salvo aquellos títulos que sigan generando importantes ventas y por tanto beneficios. En ese momento, los libros que no generan las ventas esperadas se retiran de las librerías y se sustituyen por nuevos títulos.

Por otra parte, la tirada media se está reduciendo: en 2005 se situaba en los 4.619 ejemplares y en 2016 en 2.749 , lo que ha supuesto una disminución de la tirada media en un $40,48 \%$ para el período considerado (MECD, 2017). Sin embargo, la caída en la tirada se ve compensada por la aparición de más títulos en el mercado editorial, muchos de ellos generados por el incremento del número de editoriales de pequeño tamaño. Por tanto, en el mercado editorial español se ponen en circulación más títulos, pero con menor número de ejemplares por título.

En el mercado editorial español se ponen en circulación más títulos, pero con menor número de ejemplares por título

Los grandes grupos editoriales copan la mayoría del mercado gracias a su amplia red de distribución y al elevado número de ejemplares que son capaces de producir. Sin embargo, esa producción se ha visto disminuida en los últimos tres años con reducciones en la tirada media, mientras que en las editoriales pequeñas y medianas ha ido aumentando en número con tiradas más bajas y una media de publicación de diez títulos por año.

También hay una apuesta por la edición electrónica. Desde
2008 la producción de libro en papel comenzó a caer mientras aumentaba la de libro electrónico. La edición digital supone actualmente un $28 \%$ del total de la producción editorial en España. Sin embargo, la facturación de los libros electrónicos no representa más del $5 \%$ de la facturación total, situación muy similar a la que se produce en países del entorno europeo como Francia, Alemania o Italia.

Las editoriales españolas se han visto inmersas en una caída continuada de la demanda de libros. En 2006 en España se vendían 230.626.086 ejemplares al año, diez años más tarde la cifra se sitúa en 157.233.000 (MECD, 2017).

¿Cómo explicar que el mercado editorial español haya perdido en los últimos años cerca de un $25 \%$ de facturación, pasando de los 2.933 millones de euros en 2006 a los $2.195,80$ millones de euros de 2016? Se puede achacar sólo a la crisis económica y a la piratería. España presenta elevados niveles de piratería de contenidos digitales y existe la creencia por parte de los internautas de que los contenidos online deberían ser de consumo gratuito (Ramos-Fandiño; Larrañaga-Rubio, 2011). Es evidente que la crisis económica ha conllevado un menor poder adquisitivo por parte de los consumidores y, como resultado, una ralentización en el consumo. Pero el cambio tecnológico y sus correspondientes disrupciones (Magadán-Díaz; Rivas-García, 2018) han dado lugar a cambios de hábitos de los lectores. El lector actual accede a la información y a los contenidos que tradicionalmente ha ofrecido la industria editorial de otro modo.

Todo ello (crisis económica, cambio tecnológico e innovación disruptiva y sus efectos secundarios, como la piratería y el cambio en los hábitos de lectura) ha conformado lo que podríamos catalogar de tormenta perfecta de la industria editorial.

La intención de este artículo es realizar un análisis de situación del sector editorial español que permita ver en qué medida las empresas editoriales privadas interpretan adecuadamente las señales del mercado y se adaptan al mismo. Para ello se hace uso de fuentes estadísticas oficiales disponibles sobre el mercado editorial y los hábitos de lectura, de las que se obtienen los datos necesarios para su tratamiento estadístico y se deriva un análisis econométrico que permita comprender la dinámica del mercado editorial español.

El trabajo se plantea varias preguntas para el mercado editorial español:

- ¿cómo es la elasticidad-precio de la venta de ejemplares?

- ¿qué impacto tiene el mayor o menor número de editoriales privadas sobre la venta de ejemplares?

- ¿de qué modo condiciona la venta de ejemplares la evolución de la tirada media?

- ¿qué impacto tiene el hábito de lectura en las ventas de ejemplares?

Las bases de datos de partida para el análisis econométrico son:

- Panorámica de la edición, para el período 2005-2016 (MECD, 2005-2017);

- Barómetro de hábitos de lectura y compra de libros, para el período 2012-2017;

http://federacioneditores.org/documentos.php 
- Encuesta de hábitos y prácticas culturales para los períodos 2002-2003, 2006-2007, 2010-2011 y 2014-2015.

https://www.mecd.gob.es/servicios-al-ciudadano/ estadisticas/cultura/mc/ehc/portada.html

La estructura del artículo es la siguiente: en la sección dos, se expone el marco teórico conceptual (antecedentes) que sirve de base al estudio realizado; la sección tres muestra las características de las fuentes secundarias utilizadas en el estudio; en la sección cuarta se realiza el análisis empírico partiendo de las fuentes de datos secundarias antes mencionadas; en la sección quinta se compilan los resultados obtenidos del trabajo y, finalmente, en la sección sexta se recogen las conclusiones y limitaciones derivadas de este estudio.

El presente y futuro de la industria editorial apuntan a una atomización de la oferta, gracias a las casi inexistentes barreras de entrada al mercado

\section{Antecedentes}

A través del análisis de la elasticidad-precio de la demanda de libros, se puede observar el impacto que los márgenes fijados por los intermediarios del canal de distribución tienen sobre el precio final del libro (Bittlingmayer, 1992). En gran medida dichos intermediarios al proteger sus márgenes fuerzan el mantenimiento o incluso la elevación de precios con incrementos que, en comparación con los de otros bienes de consumo, resultan mayores (Webster, 2002).

En algunos países como España son los editores quienes fijan el precio final del libro y la razón esgrimida es la de proteger los valores culturales del mercado del libro (Appelman, 2003; Van-Der-Ploeg, 2004, Canoy; Van-Ours; Van-Der-Ploeg, 2006). Sin embargo, es difícil encontrar evidencia convincente, bien teórica o empírica, de que la política de precios fijos en los libros es la mejor opción (Ringstad, 2004; Poort; Van-Eijk, 2017). Quienes se oponen a la implantación de los precios fijos señalan que la industria funciona mejor cuando opera en condiciones de libre mercado y que el precio fijo aumenta artificialmente los precios que las personas pagan por los libros (Løyland; Ringstad, 2012). Los minoristas deberían poder determinar libremente los precios de sus productos en función de la demanda real (Poort; Van-Eijk, 2017). Los oponentes a la implantación de los precios fijos en los libros creen que los gobiernos pueden apoyar mejor a la industria del libro mediante políticas que respalden la alfabetización y el desarrollo de las industrias creativas.

Tampoco hay gran cantidad de estudios acerca de la relación y grado de incidencia del precio medio del libro sobre las ventas de ejemplares, aunque sí se han tratado en la bibliografía los efectos de la dispersión de precios en internet para los ebooks (Clay; Krishnan; Wolff, 2001; Clay et al., 2002; Tang; Smith; Montgomery, 2010), la canibalización del libro usado vendido en internet sobre el libro nuevo (Ghose; Smith; Telang, 2006) o el análisis de modelos de consumo de libros por internet (De-los-Santos; Hortaçsu; Wildenbeest, 2012).
En el precio medio del ejemplar se pueden dar dos efectos contrapuestos y derivados de la reducción de costes por mejoras en los procesos productivos: por un lado, las empresas editoriales pueden mantener su tirada media de ejemplares por título y bajar el precio medio, o pueden decidir ampliar su cartera de títulos reduciendo su tirada media y mantener o, incluso, elevar el precio medio por ejemplar (Hjorth-Andersen, 2000).

En cuanto al número y dimensión de los agentes editoriales, se puede resaltar el trabajo de Szenberg y Lee (1994) sobre la estructura de la industria editorial americana que puso el foco en el hecho de que la escala mínima de producción óptima es demasiado pequeña como para suponer una barrera de entrada y evitar la existencia de un gran número de editores y excluir un alto grado de competencia. La industria editorial de publicación de libros presenta pocas barreras de entrada (Epstein, 2001; MacInnes; Kongsmak; Heckman, 2004).

La relevancia de la tirada media es analizada por Hjorth-Andersen (2000), quien subraya que ésta se ajusta para dotar al mercado de más opciones de títulos donde el lector puede escoger. Kalder (2013) y Möldre (2014), destacan el papel de la tirada media por título como indicador del estado del sector editorial en sus respectivos países (Rusia y Estonia), Ilegando a la conclusión de que la economía del libro en papel depende críticamente de la tirada media de ejemplares.

La evolución a la baja de la tirada media de ejemplares es un espejo que refleja la transición hacia un nuevo modelo de negocio editorial

Los gobiernos reconocen los beneficios de leer y apoyar las políticas que facilitan el acceso a los libros (Colbjørnsen, 2015). De hecho, la lectura tiene un efecto directo en las habilidades cognitivas del individuo (Mol; Bus, 2011; Krashen; Lee; McQuillan, 2012). El acceso a los libros también parece tener beneficios a largo plazo, como el aumento del vocabulario, el conocimiento básico y las habilidades de comprensión (Neuman; Moland, 2016).

Una mejora en la alfabetización también tiene efectos positivos sobre la economía local (Dent, 2007). La lectura y la alfabetización permiten procesar información que conducirá a la toma inteligente de decisiones (Anderson et al., 1985), algo vital en nuestra sociedad, mediante la identificación de fuentes de información, la evaluación, organización y empleo de aquella en el desarrollo de pensamiento crítico y la resolución de problemas (Hughes-Hassell; Rodge, 2007). La lectura contribuye significativamente al éxito laboral, el desarrollo profesional y la capacidad de responder al cambio (Kirsch; Guthrie, 1984).

\section{Características de las fuentes secundarias}

Este trabajo parte del análisis econométrico derivado de los datos aportados por:

- Panorámica de la edición, para el período 2005-2016; 
Tabla 1. Principales magnitudes de la edición en España (2005-2016)

\begin{tabular}{|c|c|c|c|c|c|c|}
\hline & 2005 & 2006 & 2007 & 2008 & 2009 & 2010 \\
\hline Número de agentes editoriales privados & 2.781 & 2.673 & 2.803 & 2.892 & 3.032 & 2.994 \\
\hline Número de agentes editoriales públicos & 615 & 563 & 597 & 580 & 532 & 529 \\
\hline $\begin{array}{l}\text { Porcentaje de la producción editorial de instituciones } \\
\text { públicas (títulos inscritos en ISBN) }\end{array}$ & $11,50 \%$ & $12,30 \%$ & $11,20 \%$ & $9,80 \%$ & $8,60 \%$ & $8,00 \%$ \\
\hline ISBNs concedidos & 76.265 & 77.330 & 82.559 & 104.223 & 110.205 & 114.205 \\
\hline Títulos vivos en catálogo & 325.808 & 346.706 & 369.588 & 393.012 & 414.727 & 439.991 \\
\hline Títulos editados en edición de bolsillo & 5.378 & 5.232 & 6.392 & 6.608 & 6.425 & 6.001 \\
\hline Ejemplares editados & 321.469 .155 & 338.086 .000 & 357.560 .000 & 367.460 .000 & 329.830 .000 & 302.631 .000 \\
\hline Ejemplares vendidos & 230.626 .086 & 228.220 .878 & 250.860 .500 & 240.660 .000 & 236.197 .000 & 228.230 .000 \\
\hline $\begin{array}{l}\text { Porcentaje de títulos que son traducciones de otros } \\
\text { idiomas distintos al español }\end{array}$ & $25,80 \%$ & $28,02 \%$ & $24,70 \%$ & $24,80 \%$ & $22,90 \%$ & $22,10 \%$ \\
\hline $\begin{array}{l}\text { Valor de las ventas de libros en el mercado interior } \\
\text { (millones de euros) }\end{array}$ & $2.933,20$ & $3.014,54$ & $3.123,17$ & $3.185,50$ & $3.109,58$ & $2.890,80$ \\
\hline Valor de las exportaciones de libros (miles de euros) & 452.894 & 557.052 & 554.934 & 545.982 & 442.367 & 457.792 \\
\hline $\begin{array}{l}\text { Tirada media de ejemplares por título (número de } \\
\text { ejemplares) }\end{array}$ & 4.619 & 4.905 & 5.475 & 5.035 & 4.328 & 3.790 \\
\hline Precio medio del libro (euros) & 12,72 & 13,21 & 11,75 & 13,26 & 13,17 & 12,67 \\
\hline
\end{tabular}

Fuente: elaborado a partir de la Panorámica de la edición en España

- Barómetro de hábitos de lectura y compra de libros, para el período 2012-2017;

- Encuesta de hábitos y prácticas culturales para los períodos 2002-2003, 2006-2007, 2010-2011 y 2014-2015.

En la tabla 1 se recogen las principales magnitudes de la edición en España empleadas para llevar a cabo el análisis econométrico con objeto de contrastar los resultados con los datos derivados de las encuestas que analizan los hábitos de lectura en España.
De modo resumido, en la tabla 2 se recogen los datos principales de la ficha técnica y metodológica del Barómetro de hábitos de lectura y compra de libros.

A esta información debe añadirse la categorización efectuada por el mismo documento en relación con los lectores, tal y como se resume en la tabla 3.

En relación con la Encuesta de hábitos y prácticas culturales, de modo sintético se expone en la tabla 4 la metodología y características de las encuestas oficiales empleadas.

Tabla 1 (cont.). Principales magnitudes de la edición en España (2005-2016)

\begin{tabular}{|c|c|c|c|c|c|c|}
\hline & 2011 & 2012 & 2013 & 2014 & 2015 & 2016 \\
\hline Número de agentes editoriales privados & 2.988 & 2.835 & 2.750 & 2.786 & 2.649 & 2.722 \\
\hline Número de agentes editoriales públicos & 476 & 352 & 336 & 323 & 314 & 304 \\
\hline $\begin{array}{l}\text { Porcentaje de la producción editorial de instituciones } \\
\text { públicas (títulos inscritos en ISBN) }\end{array}$ & $7,00 \%$ & $7,50 \%$ & $9,00 \%$ & $9,00 \%$ & $10,10 \%$ & $9,60 \%$ \\
\hline ISBNs concedidos & 111.907 & 104.724 & 89.130 & 90.802 & 79.397 & 86.000 \\
\hline Títulos vivos en catálogo & 464.981 & 490.752 & 524.213 & 553.884 & 586.811 & 620.316 \\
\hline Títulos editados en edición de bolsillo & 5.376 & 4.806 & 4.099 & 3.615 & 4.211 & 4.471 \\
\hline Ejemplares editados & 286.462 .000 & 280.251 .000 & 246.345 .000 & 226.590 .000 & 225.277 .000 & 224.054 .000 \\
\hline Ejemplares vendidos & 199.814 .000 & 170.237 .000 & 153.830 .000 & 153.620 .000 & 155.436 .000 & 157.233 .000 \\
\hline $\begin{array}{l}\text { Porcentaje de títulos que son traducciones de otros } \\
\text { idiomas distintos al español }\end{array}$ & $21,10 \%$ & $22,00 \%$ & $22,30 \%$ & $21,20 \%$ & $20,60 \%$ & $16,10 \%$ \\
\hline $\begin{array}{l}\text { Valor de las ventas de libros en el mercado interior } \\
\text { (millones de euros) }\end{array}$ & $2.772,34$ & $2.471,49$ & $2.181,97$ & $2.195,80$ & $2.257,67$ & $2.317,20$ \\
\hline Valor de las exportaciones de libros (miles de euros) & 506.537 & 527.343 & 526.482 & 541.765 & 522.366 & 571.767 \\
\hline $\begin{array}{l}\text { Tirada media de ejemplares por título (número de } \\
\text { ejemplares) }\end{array}$ & 3.441 & 3.540 & 3.223 & 2.886 & 2.810 & 2.749 \\
\hline Precio medio del libro (euros) & 13,87 & 14,52 & 14,18 & 14,29 & 14,52 & 14,74 \\
\hline
\end{tabular}

Fuente: elaborado a partir de la Panorámica de la edición en España 
Tabla 2. Ficha técnica de la encuesta del Barómetro de hábitos de lectura y compra de libros

\begin{tabular}{|c|c|c|}
\hline Concepto & Encuesta 2011 & Encuesta 2017 \\
\hline Universo & $\begin{array}{l}\text { Individuos de más de } 10 \text { años residentes en la España } \\
\text { peninsular, Islas Baleares e Islas Canarias. }\end{array}$ & $\begin{array}{l}\text { Individuos de más de } 10 \text { años residentes en la España } \\
\text { peninsular, Islas Baleares e Islas Canarias. }\end{array}$ \\
\hline Ámbito & $\begin{array}{l}\text { Nacional, con representación de todas las regiones y } \\
\text { tamaños de hábitat. }\end{array}$ & $\begin{array}{l}\text { Nacional, con representación de todas las regiones y } \\
\text { tamaños de hábitat. }\end{array}$ \\
\hline Metodología & $\begin{array}{l}\text { Entrevista telefónica asistida por ordenador (CATI), a una } \\
\text { muestra mixta de teléfonos fijos y teléfonos móviles. }\end{array}$ & $\begin{array}{l}\text { Entrevista telefónica asistida por ordenador (CATI), a una } \\
\text { muestra mixta de teléfonos fijos y teléfonos móviles. }\end{array}$ \\
\hline Técnica de muestreo & $\begin{array}{l}\text { Mixta. Aleatorio estratificado por Comunidad Autónoma y } \\
\text { tamaño de hábitat, con afijación no proporcional por co-- } \\
\text { munidad autónoma y proporcional por provincia y tamaño } \\
\text { de hábitat dentro de cada comunidad. }\end{array}$ & $\begin{array}{l}\text { Mixta. Aleatorio estratificado por Comunidad Autónoma } \\
\text { y tamaño de hábitat, con afijación no proporcional por } \\
\text { comunidad autónoma y proporcional por provincia y } \\
\text { tamaño de hábitat dentro de cada comunidad. }\end{array}$ \\
\hline Tamaño de la muestra & 6.700 entrevistas & 5.000 entrevistas \\
\hline Error muestral & $\begin{array}{l}\text { Para el total de la muestra: } \pm 1,22 \% \\
\text { Por comunidades autónomas el error muestral va desde el } \\
\pm 3,5 \% \text { en Andalucía al } \pm 6,9 \text { en La Rioja. }\end{array}$ & $\begin{array}{l}\text { Para el total de la muestra: } \pm 1,41 \% \\
\text { Por comunidades autónomas el error muestral va desde el } \\
\pm 4,3 \% \text { en Andalucía al } \pm 7,3 \text { en La Rioja. }\end{array}$ \\
\hline Cuestionario & $\begin{array}{l}\text { Estructurado con preguntas cerradas precodificadas y } \\
\text { alguna pregunta abierta, con una duración máxima de } 20 \\
\text { minutos. }\end{array}$ & $\begin{array}{l}\text { Estructurado con preguntas cerradas precodificadas y } \\
\text { alguna pregunta abierta, con una duración máxima de } 20 \\
\text { minutos. }\end{array}$ \\
\hline Momento de realización & Último trimestre de 2011. & Último trimestre de 2017. \\
\hline
\end{tabular}

Fuente: elaborado a partir del Barómetro de hábitos de lectura y compra de libros (2011-2017)

Tabla 3. Tipología de lectores empleada en el Barómetro de hábitos de lectura y compra de libros

\begin{tabular}{|l|l|}
\hline Tipo & Definición \\
\hline Lectores frecuentes & Leen, al menos, una vez a la semana. \\
\hline Lectores habituales & Conjunto formado por los lectores frecuentes y aquellos que leen, al menos, una vez al mes. \\
\hline Lector ocasional & Conjunto formado por los lectores habituales, excluidos los frecuentes, y aquellos que leen, al menos, una vez al trimestre. \\
\hline No lector & No leen nunca o casi nunca. \\
\hline
\end{tabular}

Fuente: elaborado a partir del Barómetro de hábitos de lectura y compra de libros (2011-2017)

\section{Análisis y resultados}

\subsection{Venta de libros y precio medio por ejemplar}

La Ley 10/2007, de 22 de junio, de la lectura, del libro y de las bibliotecas (España, 2007) tiene como finalidad ensalzar el valor del libro, el proceso lector e instaurar principios que regulen el comercio del libro. El Artículo 9 de la citada Ley desarrolla la regulación respecto al precio del libro, que está sujeto a un precio fijo de venta al público que deben acatar toda la cadena de valor: el editor, el distribuidor y la librería.

El precio medio del libro se puede calcular aproximativamente a partir de la ratio entre facturación y ejemplares

Tabla 4. Ficha técnica de la Encuesta de hábitos y prácticas culturales

\begin{tabular}{|c|c|c|c|c|}
\hline Concepto & 2002-2003 & 2006-2007 & 2010-2011 & 2014-2015 \\
\hline Universo & $\begin{array}{l}\text { Población de } 15 \text { años y más } \\
\text { que reside en viviendas } \\
\text { familiares. }\end{array}$ & $\begin{array}{l}\text { Población de } 15 \text { años y más } \\
\text { que reside en viviendas } \\
\text { familiares. }\end{array}$ & $\begin{array}{l}\text { Población de } 15 \text { años y más } \\
\text { que reside en viviendas } \\
\text { familiares. }\end{array}$ & $\begin{array}{l}\text { Población de } 15 \text { años y más } \\
\text { que reside en viviendas } \\
\text { familiares. }\end{array}$ \\
\hline Ámbito & $\begin{array}{l}\text { Territorio nacional, con ex- } \\
\text { cepción de Ceuta y Melilla. }\end{array}$ & $\begin{array}{l}\text { Territorio nacional, con ex- } \\
\text { cepción de Ceuta y Melilla. }\end{array}$ & $\begin{array}{l}\text { Territorio nacional, con ex- } \\
\text { cepción de Ceuta y Melilla. }\end{array}$ & $\begin{array}{l}\text { Territorio nacional, con ex- } \\
\text { cepción de Ceuta y Melilla. }\end{array}$ \\
\hline Metodología & Entrevista personal & Entrevista personal & Entrevista personal & Entrevista personal \\
\hline Técnica de muestreo & Polietápico estratificado & Polietápico estratificado & Polietápico estratificado & Polietápico estratificado \\
\hline $\begin{array}{l}\text { Tamaño de la } \\
\text { muestra }\end{array}$ & 12.180 & 16.400 & 16.400 & 16.500 \\
\hline Error muestral & $\pm 1 \%$ & $\pm 0,42 \%$ & $\pm 0,43 \%$ & $\pm 0,43 \%$ \\
\hline Cuestionario & $\begin{array}{l}\text { Estructurado con preguntas } \\
\text { cerradas precodificadas y } \\
\text { alguna pregunta abierta }\end{array}$ & $\begin{array}{l}\text { Estructurado con preguntas } \\
\text { cerradas precodificadas y } \\
\text { alguna pregunta abierta }\end{array}$ & $\begin{array}{l}\text { Estructurado con preguntas } \\
\text { cerradas precodificadas y } \\
\text { alguna pregunta abierta }\end{array}$ & $\begin{array}{l}\text { Estructurado con preguntas } \\
\text { cerradas precodificadas y } \\
\text { alguna pregunta abierta }\end{array}$ \\
\hline $\begin{array}{l}\text { Momento de } \\
\text { realización }\end{array}$ & $\begin{array}{l}\text { Segundo, tercer y cuarto } \\
\text { trimestre de } 2002 \text { y primer } \\
\text { trimestre de } 2003 \text {. }\end{array}$ & $\begin{array}{l}\text { De marzo de } 2006 \text { a febrero } \\
\text { de } 2007 .\end{array}$ & $\begin{array}{l}\text { De marzo de } 2010 \text { a febrero } \\
\text { de } 2011 .\end{array}$ & $\begin{array}{l}\text { De marzo de } 2014 \text { a febrero } \\
\text { de } 2015 \text {. }\end{array}$ \\
\hline
\end{tabular}

Fuente: elaborado a partir del Encuesta de hábitos y prácticas culturales (2002-2015) 
vendidos por la editorial. Por soportes, aumenta el precio del libro en papel y desciende el del libro en otros soportes, particularmente el del libro digital. El precio del libro digital se sitúa en España, de media, 10 euros por debajo del precio medio del libro en papel.

La primera pregunta que se formula en este estudio tiene que ver con el impacto de los precios del libro sobre las ventas. Para dar respuesta a la misma, se plantea una sencilla ecuación en donde $\mathrm{V}$ representa la venta anual de ejemplares en el mercado interior en millones de euros, y $\mathrm{P}$ es el precio medio por ejemplar, expresado en euros:

$$
V=\alpha_{0}+\alpha_{1} P+\varepsilon
$$

Los resultados generados por la regresión lineal se resumen en las tablas 5 y 6 que muestran tanto la fiabilidad de la ecuación como la significatividad de la relación establecida.

La tabla 5 muestra los parámetros empleados para evaluar la bondad de ajuste que, en este caso, es relativamente aceptable: la variabilidad de $V$ se explica por $P$ en un $68,9 \%$. En resumen, la variable precio explica razonablemente el comportamiento de las ventas de libros.

La tabla 6 ofrece los datos concretos de los valores de los parámetros y la prueba t de Student de cada uno de ellos.

Se observa claramente la relación negativa existente entre $\mathrm{V}$ y $\mathrm{P}$ y la significatividad de los parámetros determinados. De todo ello se puede especificar la expresión [1] analítica como:

$$
V=7493002912-352748772 P
$$

rantiza que sus productos sean sustitutivos lejanos, lo que se traduciría en una reducción de la elasticidad del precio de las ventas.

\subsection{Venta de libros y negocio editorial}

En los últimos años el sector está experimentando un importante descenso en su cifra de negocio. La crisis económica y financiera iniciada en 2007 y, consecuentemente, la reducción del consumo y de la demanda interna parece, tal y como apunta la Federación de Gremios de Editores de España (FGEE), la causa principal de esta bajada. Una crisis cuyos efectos no se hicieron patentes hasta 2009, cuando se registró el primer retroceso $(-2,4 \%)$, y a la que se han ido sumando otros factores como:

- incertidumbre provocada por la reforma educativa;

- el proceso de cambio en los hábitos de consumo cultural, como consecuencia de la evolución digital.

La segunda pregunta que se plantea en este trabajo es la relativa al impacto que tiene el mayor o menor número de editoriales privadas sobre la venta de ejemplares. Para encontrar una respuesta, se partirá de una expresión básica
En el gráfico 1 se muestra la representación de esta relación entre ventas de ejemplares y precio medio.

Estamos ante una función de pendiente negativa, elástica (su elasticidad en los diversos puntos considerados se mueve en el intervalo [1,27-2,2]), lo que confirma que los "productos" editoriales son sustitutivos cercanos (Bittlingmayer, 1992; Webster, 2002).

A pesar de que en España el precio por edición de un libro es fijo y sólo puede modificarse por nuevas reimpresiones o ediciones, esta variable afecta a la facturación de las empresas de modo significativo (Appelman, 2003; Van-DerPloeg, 2004, Canoy; Van-Ours; Van-DerPloeg, 2006).

Por tal razón, la capacidad de las editoriales para disponer de las obras en exclusiva de determinados autores les ga-

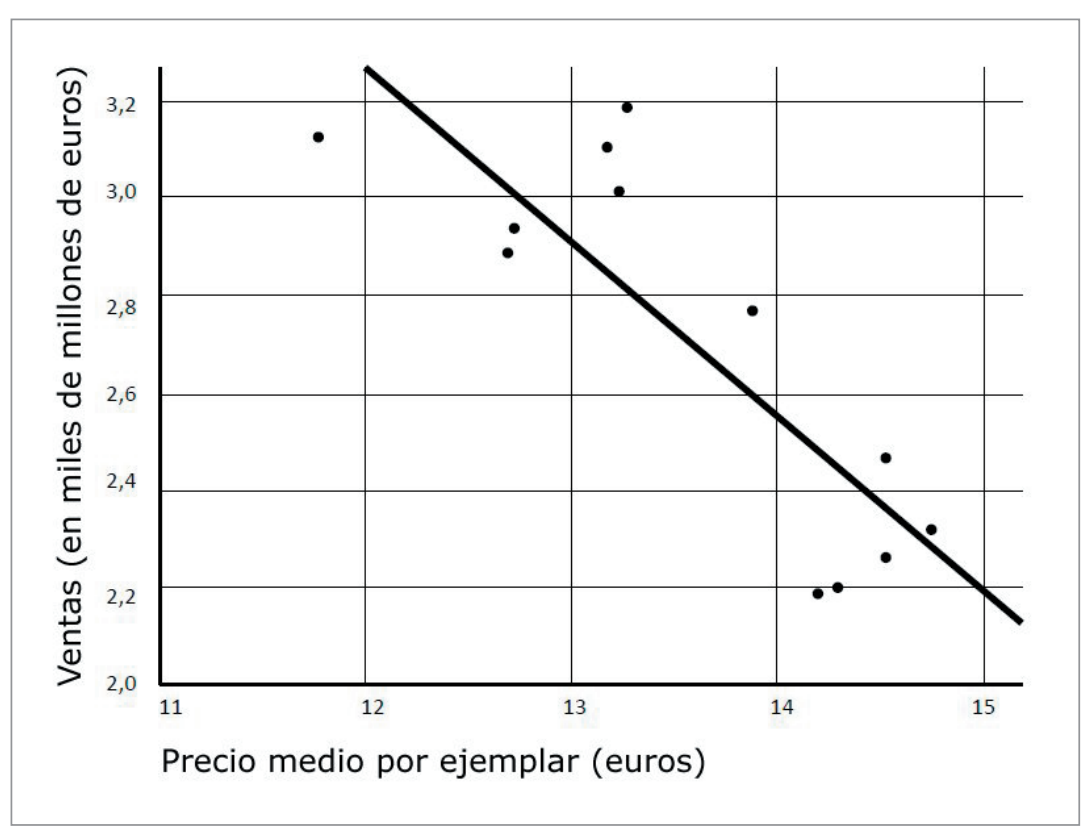

Gráfico 1. Relación ventas-precio 
Tabla 7. Resumen de modelo y estimaciones de parámetro

\begin{tabular}{|l|c|c|c|c|c|c|}
\hline \multirow{2}{*}{ Ecuación } & \multicolumn{4}{|c|}{ Resumen del modelo } & \multicolumn{2}{c|}{ Estimaciones de parámetro } \\
\cline { 2 - 7 } & R cuadrado & F & gl1 & gl2 & b1 \\
\hline Lineal & 0,975 & 433,480 & 1 & 11 & 0,000 & $103.514,873$ \\
\hline
\end{tabular}

que se analiza econométricamente: la relación entre ejemplares vendidos anualmente en el mercado interior $(L)$-en número de copias- y número de editoriales privadas $(\mathrm{E})$ :

$$
L=\beta E+\varepsilon
$$

Los resultados generados por la regresión se resumen en la tabla 7 y muestran tanto la fiabilidad de la ecuación como la significatividad de la relación establecida.

La tabla 7 nos muestra los diferentes parámetros empleados para evaluar la bondad de ajuste que, en este caso, es muy aceptable: la variabilidad de $V$ se explica por $E$ en un 97,5 por 100. Así, la expresión [3] se representaría analítica y gráficamente como sigue:

$$
L=103.514,9 . E
$$

Por tanto, se deduce que a mayor número de agentes editores, mayor es el nivel de ventas de libros. La relación que existe en el sector editorial español entre ventas de ejemplares y número de empresas editoriales es la mostrada en el gráfico 2.

Se deriva de este sencillo análisis que si durante el período 2005-2016 la media de ejemplares por título fue de 3.900 copias (cálculo derivado de la tabla 1), entonces, considerando el parámetro de [4], el número medio de títulos producidos por empresa editorial al año es de 26-27 títulos. Obviamente los grandes grupos editoriales producen muchos más títulos, pero los datos y el análisis econométrico apuntan a:

- una atomización de la oferta, con sellos editoriales que buscan el micro-espacio del mercado que les permita desarrollar su actividad gracias a las casi inexistentes barreras de entrada (Szenberg; Lee, 1994; Epstein, 2001; MacInnes; Kongsmak; Heckman, 2004);

- un posible estancamiento de la producción editorial de los grandes grupos, lo que se podría compensar, aunque sólo parcialmente, por un esfuerzo exportador mayor.

\subsection{Tirada media y ventas}

La tirada de una obra constituye el resultado del cálculo que el editor efectúa acerca de la acogida potencial de esta en el mercado, es una representación de las expectativas de colocación que se le suponen. Cuanto más tímidas son las tiradas menos posibilidades de venta se les atribuyen y viceversa (Cordón-García, 2002).

La tercera cuestión planteada tiene que ver con la influencia que la venta de ejemplares tiene sobre la decisión de alterar la tirada media.

La expresión básica que se analiza econométricamente es la de tirada media de ejemplares de un momento temporal $t\left(T_{t}\right)$ y los ejemplares vendidos en un momento temporal anterior, $\mathrm{t}-1\left(\mathrm{~L}_{\mathrm{t}-1}\right)$ :

$$
T_{t}=\gamma L_{t-1}+\varepsilon
$$

Los resultados generados por el análisis se resumen en la tabla 8 y muestran tanto la fiabilidad de la ecuación como la significatividad de la relación establecida. A mayor nivel de ventas mayor tirada y viceversa.

La tabla 8 muestra los parámetros empleados para evaluar la bondad de ajuste en los modelos econométricos analizados y que en todos los casos estudiados es muy aceptable: la variabilidad de $T$ se explica por $L$ en un intervalo que oscila entre el $80,8 \%$ y el $87,9 \%$.

Tabla 8. Resumen del modelo

\begin{tabular}{|c|c|c|c|}
\hline $\mathbf{R}$ & R cuadrado & $\begin{array}{c}\text { R cuadrado } \\
\text { ajustado }\end{array}$ & $\begin{array}{c}\text { Error estándar } \\
\text { de la estimación }\end{array}$ \\
\hline 0,991 & 0,982 & 0,980 & 556,394 \\
\hline
\end{tabular}

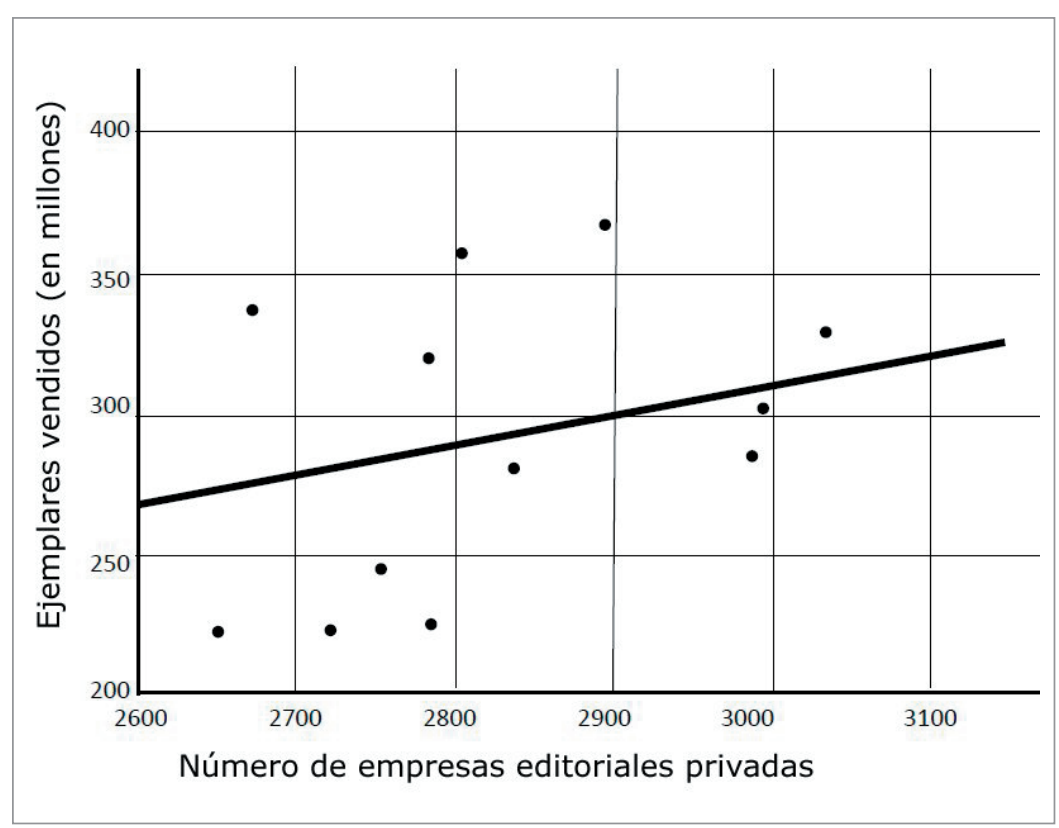

Gráfico 2. Relación ventas-empresas 
Tabla 9. Coeficiente y significatividad t

\begin{tabular}{|l|c|c|c|c|c|}
\cline { 2 - 5 } \multicolumn{1}{c|}{} & \multicolumn{2}{|c|}{ Coeficientes no estandarizados } & $\begin{array}{c}\text { Coeficientes } \\
\text { estandarizados }\end{array}$ & \multirow{2}{*}{ t } & \multirow{2}{*}{ Sig. } \\
\cline { 2 - 4 } & B & Desv. error & Beta & \\
\hline $\begin{array}{l}\text { Venta de } \\
\text { ejemplares }\end{array}$ & $1,882 \mathrm{E}-5$ & 0,000 & 0,991 & 23,291 & 0,000 \\
\hline
\end{tabular}

La tabla 9 muestra los valores del coeficiente y su significatividad.

La expresión [5] se concretaría como:

$$
T_{t}=188.200 L_{t-1}
$$

Y su representación gráfica se muestra en el gráfico 3.

De los datos obtenidos se deriva que las empresas editoriales adaptan las tiradas medias por título en un período en función de los ejemplares vendidos en el mercado en el período anterior. Se confirma así la relevancia de la decisión de la tirada media (Hjorth-Andersen, 2000) y cómo se ajusta en función de las ventas realizadas (Kalder, 2013; Möldre, 2014).

Las empresas editoriales adaptan las tiradas medias por título en un período en función de los ejemplares vendidos en el período anterior

\subsection{Ejemplares vendidos y hábitos de lectura}

La última hipótesis que se considera es el impacto significativo del hábito de lectura sobre la venta de ejemplares en el mercado nacional.

Se supone una relación econométrica del tipo:

$$
L=\delta H+\varepsilon
$$

Donde $L$ es el número de ejemplares vendidos y $\mathrm{H}$ es el número de lectores habituales. Los datos del análisis econométrico son los reflejados en la tabla 10 y la representación se recoge en el gráfico 4.

La expresión analítica quedaría del siguiente modo:

$$
L=6,905 \cdot H+\varepsilon
$$

Aunque en una primera aproximación pueda parecer que se confirma la existencia de una relación positiva y directa entre las ventas de ejemplares y el número de lectores habituales, esto puede deberse más a la simplificación operada por el modelo lineal sobre la estructura y distribución de los datos. Una reflexión más pausada permite intuir que no todos los lectores habituales deberían ser necesariamente compradores de libros en el mercado editorial: se lee más de lo que efectivamente se vende.

Las políticas públicas de promoción y acceso a los libros tienen su efecto social pero no impactan todo lo que sería deseable en la industria editorial

Se concluye que las políticas públicas de promoción y acceso a los libros tienen su efecto social (Colbjørnsen, 2015; Neuman; Moland, 2016) pero no impacta todo lo que sería deseable en el mercado editorial.

\section{Conclusiones y limitaciones}

A través de este estudio aproximativo al mercado editorial español, se ha podido avanzar una serie de respuestas a las preguntas formuladas inicialmente. Todo ello resalta la complejidad y dinamismo al que se enfrentan las empresas del mercado editorial, que están actualmente en pleno proceso de adaptación de sus respectivos modelos de negocio al cambio técnico (Magadán-Díaz; Rivas-García, 2018).

Resulta reveladora la dinámica de la industria editorial española, la cuarta potencia editorial a nivel mundial, que se ajusta a un patrón anticipado por los diversos estudios señalados en la revisión de los antecedentes en relación con los precios, las empresas y las tiradas. Sin embargo, el hábito de lectura podría haber supuesto un mayor impacto en el mercado editorial del que de modo efectivo tiene. Quizá la crisis económica y su impacto sobre las economías domésticas han tenido que ver en esa relación menos estrecha de lo esperada. Sería un tema a tratar más profundamente en trabajos posteriores.

Gráfico 3. Relación tirada-ventas 
Tabla 10. Resumen de modelo y estimación de parámetro

\begin{tabular}{|l|c|c|c|c|c|c|}
\hline \multirow{2}{*}{ Ecuación } & \multicolumn{4}{|c|}{ Resumen del modelo } & \multicolumn{2}{c|}{ Estimaciones de parámetro } \\
\cline { 2 - 7 } & R cuadrado & F & gl1 & gl2 & Sig. & b1 \\
\hline Lineal & 0,946 & 192,780 & 1 & 11 & 0,000 & 6,905 \\
\hline
\end{tabular}

En el análisis ofrecido no se ha tenido en cuenta el papel del ebook en el mercado editorial español, todavía reducido, pero será necesario abordar un estudio más amplio que integre esta línea de negocio valorando aspectos análogos a los considerados en este trabajo. Tampoco se ha considerado el papel de las instituciones públicas como editoras o de otras figuras editoriales informales (por ejemplo, la figura del autor-editor) y el peso que podrían tener de cara al futuro panorama de la edición en España.

\section{Referencias}

Anderson, Richard C.; Hiebert, Elfrieda; Scott, Judith A.; Wilkinson, Ian (1985). Becoming a nation of readers: The report of the Commission on Reading. Washington, DC: University of Illinois, Becoming a Nation of Readers National Inst. of Education (ED).

http://textproject.org/assets/library/resources/AndersonHiebert-Scott-Wilkinson-Becoming-a-Nation-of-Readers.pdf

Appelman, Marja (2003). "Fixed book price". In: Towse, R. A handbook of cultural economics. Cheltenham, UK: Edward Elgar Publishing Limited, pp. 237-242.

https://core.ac.uk/download/pdf/18507444.pdf

Bittlingmayer, George (1992). "The elasticity of demand for books, resale price maintenance and the lerner index". Journal of institutional and theoretical economics (JITE) / Zeitschrift für die gesamte Staatswissenschaft, v. 148, n. 4, pp. 588-606.

https://www.jstor.org/stable/40751553

Canoy, Marcel; Van-Ours, Jan; Van-Der-Ploeg, Frederick (2006). "The economics of books". Handbook of the economics of art and culture, v. 1, pp. 721-761.

https://doi.org/10.1016/S1574-0676(06)01021-0

Clay, Karen; Krishnan, Ramayya; Wolff, Eric (2001). "Prices and price dispersion on the Web: Evidence from the online book industry". Journal of industrial economics, v. 49, n. 4, pp. 521-539.

http://www.nber.org/papers/w8271

https://doi.org/10.1111/1467-6451.00161

Clay, Karen; Krishnan, Ramayya; Wolff, Eric; Fernandes, Danny (2002). "Retail strategies on the Web: Price and non-price competition in the online book industry". Journal of industrial economics, v. 50, n. 3, pp. 351-367.

https://goo.gl/eXMXJt

https://doi.org/10.1111/1467-6451.00181

Colbjørnsen, Terje (2015). "What is the VAT? The policies

and practices of value added tax on ebooks in Europe". International journal of cultural policy, v. 21, n. 3, pp. 326-343. https://doi.org/10.1080/10286632.2014.904298

Cordón-García, José-Antonio (2002). "La edición en España". Sintagma: Revista de historia del libro y de la lectura, v. 1, pp. 113-136.

http://hdl.handle.net/10760/15081

De-los-Santos, Babur; Hortaçsu, Ali; Wildenbeest, Matthijs (2012). "Testing models of consumer search using data on web browsing and purchasing behavior". American economic review, v. 102, n. 6, pp. 2955-2980.

https://kelley.iu.edu/mwildenb/searchtest.pdf https://doi.org/10.1257/aer.102.6.2955

Dent, Valeda (2007). "Local economic development in Uganda and the connection to rural community libraries and literacy". New library world, v. 108, n. 5-6, pp. 203-217. https://rucore.libraries.rutgers.edu/rutgers-lib/21707/ PDF/1/play

https://doi.org/10.1108/03074800710748777

Epstein, Jason (2001). Book business. Boston: Norton and Company. ISBN: 9780393322347

España (2007). "Ley 20/2007, de 22 de junio, de la lectura, del libro y de las bibliotecas". BOE, n. 150, 23 junio.

https://www.boe.es/boe/dias/2007/06/23/pdfs/A2714027150.pdf

Ghose, Anindya; Smith, Michael; Telang, Rahul (2006). Internet exchanges for used books: An empirical analysis of product cannibalization and welfare impact. Information systems research, v. 17, n. 1, pp. 3-19.

http://pages.stern.nyu.edu/ aghose/UsedBook.pdf 
https://doi.org/10.1287/isre.1050.0072

Hjorth-Andersen, Christian (2000). "A model of the Danish book market". Journal of cultural economics, v. 24, n. 1, pp. 27-43.

https://bit.ly/2q/VpvL

https://doi.org/10.1023/A:1007514320221

Hughes-Hassell, Sandra; Rodge, Pradnya (2007). "The leisure reading habits of urban adolescents". Journal of adolescent and adult literacy, v. 51, n. 1, pp. 22-33.

https://doi.org/10.1598/JAAL.51.1.3

Kalder, Daniel (2013). "Notes towards a complete taxonomy of murkiness: publishing in Russia". Publishing research quarterly, v. 29, n. 2, pp. 151-163.

https://doi.org/10.1007/s12109-013-9316-5

Kirsch, Irwin; Guthrie, John (1984). "Adult reading practices for work and leisure". Adult education quarterly, v. 34, n. 4, pp. 213-232.

https://doi.org/10.1177/0001848184034004003

Krashen, Stephen; Lee, Syying; McQuillan, Jeff (2012). “Is the library important? Multivariate studies at the national and international level". Journal of language and literacy education, v. 8, n. 1, pp. 26-38.

http://jolle.coe.uga.edu/wp-content/uploads/2012/06/Isthe-Library-Important.pdf

Løyland, Knut; Ringstad, Vidar (2012). "Fixed or free book prices: is a hybrid system superior?". International journal of cultural policy, v. 18, n. 2, pp. 238-254.

https://doi.org/10.1080/10286632.2011.573850

Maclnnes, Ian; Kongsmak, Kasama; Heckman, Robert (2004). "Vertical integration and the relationship between publisher and creators". Journal of electronic commerce research, v. 5, n. 1, pp. 25-37.

http://web.csulb.edu/journals/jecr/issues/20041/Paper3.pdf

Magadán-Díaz, Marta; Rivas-García, Jesús (2018). “Digitization and business models in the Spanish publishing industry". Publishing research quarterly, v. 34, n. 3, pp. 333-346. https://doi.org/10.1007/s12109-018-9593-0

Milliot, Jim (2017). "The world's 54 largest publishers, 2017". Publisher weekly, August 25 $5^{\text {th }}$.

https://bit.ly/2yG50Ci

MECD (2005). Panorámica de la edición española de libros 2004. Análisis sectorial del libro. Madrid: Ministerio de Educación, Cultura y Deporte, Secretaría General Técnica.

http://sede.educacion.gob.es/publiventa/detalle.action?cod=12306C

MECD (2006). Panorámica de la edición española de libros 2005. Análisis sectorial del libro. Madrid: Ministerio de Educación, Cultura y Deporte, Secretaría General Técnica. http://sede.educacion.gob.es/publiventa/detalle.action?cod=12675C

MECD (2007). Panorámica de la edición española de libros 2006. Análisis sectorial del libro. Madrid: Ministerio de Educación, Cultura y Deporte, Secretaría General Técnica. http://sede.educacion.gob.es/publiventa/detalle.action?cod=12958C

MECD (2008). Panorámica de la edición española de libros 2007. Análisis sectorial del libro. Madrid: Ministerio de Edu- cación, Cultura y Deporte, Secretaría General Técnica. http://sede.educacion.gob.es/publiventa/detalle.action?cod=13259C

MECD (2009). Panorámica de la edición española de libros 2008. Análisis sectorial del libro. Madrid: Ministerio de Educación, Cultura y Deporte, Secretaría General Técnica. http://sede.educacion.gob.es/publiventa/detalle.action?cod=13556C

MECD (2010). Panorámica de la edición española de libros 2009. Análisis sectorial del libro. Madrid: Ministerio de Educación, Cultura y Deporte, Secretaría General Técnica. http://sede.educacion.gob.es/publiventa/detalle.action?cod=13784C

MECD (2011). Panorámica de la edición española de libros 2010. Análisis sectorial del libro. Madrid: Ministerio de Educación, Cultura y Deporte, Secretaría General Técnica. http://sede.educacion.gob.es/publiventa/detalle.action?cod=13997C

MECD (2012). Panorámica de la edición española de libros 2011. Análisis sectorial del libro. Madrid: Ministerio de Educación, Cultura y Deporte, Secretaría General Técnica. http://sede.educacion.gob.es/publiventa/detalle.action?cod=14236C

MECD (2013). Panorámica de la edición española de libros 2012. Análisis sectorial del libro. Madrid: Ministerio de Educación, Cultura y Deporte, Secretaría General Técnica.

http://sede.educacion.gob.es/publiventa/detalle.action?cod=14452C

MECD (2014). Panorámica de la edición española de libros 2013. Análisis sectorial del libro. Madrid: Ministerio de Educación, Cultura y Deporte, Secretaría General Técnica. http://sede.educacion.gob.es/publiventa/detalle.action?cod=20009C

MECD (2015). Panorámica de la edición española de libros 2014. Análisis sectorial del libro. Madrid: Ministerio de Educación, Cultura y Deporte, Secretaría General Técnica.

http://sede.educacion.gob.es/publiventa/detalle.action?cod=20199C

MECD (2017). Panorámica de la edición española de libros 2016. Análisis sectorial del libro. Madrid: Ministerio de Educación, Cultura y Deporte, Secretaría General Técnica.

http://sede.educacion.gob.es/publiventa/detalle.action?cod=21061C

Mol, Suzanne; Bus, Adriana (2011). "To read or not to read: A meta-analysis of print exposure from infancy to early adulthood". Psychological bulletin, v. 137, n. 2, pp. 267-296. https://goo.gl/spDUpx https://doi.org/10.1037/a0021890

Möldre, Aile (2014). "Estonian publishing sector at the turn of the $20^{\text {th }}$ and $21^{\text {st }}$ centuries: Emergence of e-book market". Knygotyra, v. 62, pp. 94-112.

http://www.zurnalai.vu.It/knygotyra/article/viewFile/3607/2619

Neuman, Susan; Moland, Naomi (2016). "Book deserts: The consequences of income segregation on children's access to print". Urban education, v. 1, pp. 1-22.

http://detroitlittlelibraries.org/wp-content/uploads/2016/07/ BookDesertsStudy.pdf https://doi.org/10.1177/0042085916654525

Poort, Joost; Van-Eijk, Nico (2017). "Digital fixation: The law and economics of a fixed e-book price. International journal of cultural policy, v. 23, n.4, pp. 464-481. https://doi.org/10.1080/10286632.2015.1061516

Ramos-Fandiño, Guadalupe-Patricia; Larrañaga-Rubio, Ju- 
lio (2011). “La difusión y uso del ebook en España desde una perspectiva socioeconómica". Revista general de información y documentación, v. 21, pp. 39-52. https://doi.org/10.5209/rev_RGID.2011.v21.37428

Ringstad, Vidar (2004). "On the cultural blessings of fixed book prices: facts or fiction?" International journal of cultural policy, v. 10, n. 3, pp. 351-365.

https://doi.org/10.1080/1028663042000312570

Szenberg, Michael; Lee, Eric-Youngkoo (1994). "The structure of the American book publishing industry". Journal of cultural economics, v. 18, n. 4, pp. 313-322.

https://doi.org/10.1007/BF01079762

Tang, Zhulei; Smith, Michael; Montgomery, Alan (2010).
"The impact of shopbot use on prices and price dispersion: Evidence from online book retailing". International journal of industrial organization, v. 28, n. 6, pp. 579-590.

https://doi.org/10.1016/j.ijindorg.2010.03.014

Van-Der-Ploeg, Frederick (2004). "Beyond the dogma of the fixed book price agreement". Journal of cultural economics, v. 28 , n. 1 , pp. 1-20.

https://goo.gl/qhahy4

https://doi.org/10.1023/B:JCEC.0000009817.61107.f3

Webster, Thomas (2002). "Retail consolidation and the price elasticity of demand for books". International business and economics research journal, v. 1, n. 2, pp. 27-34. https://doi.org/10.19030/iber.v1i12.4005
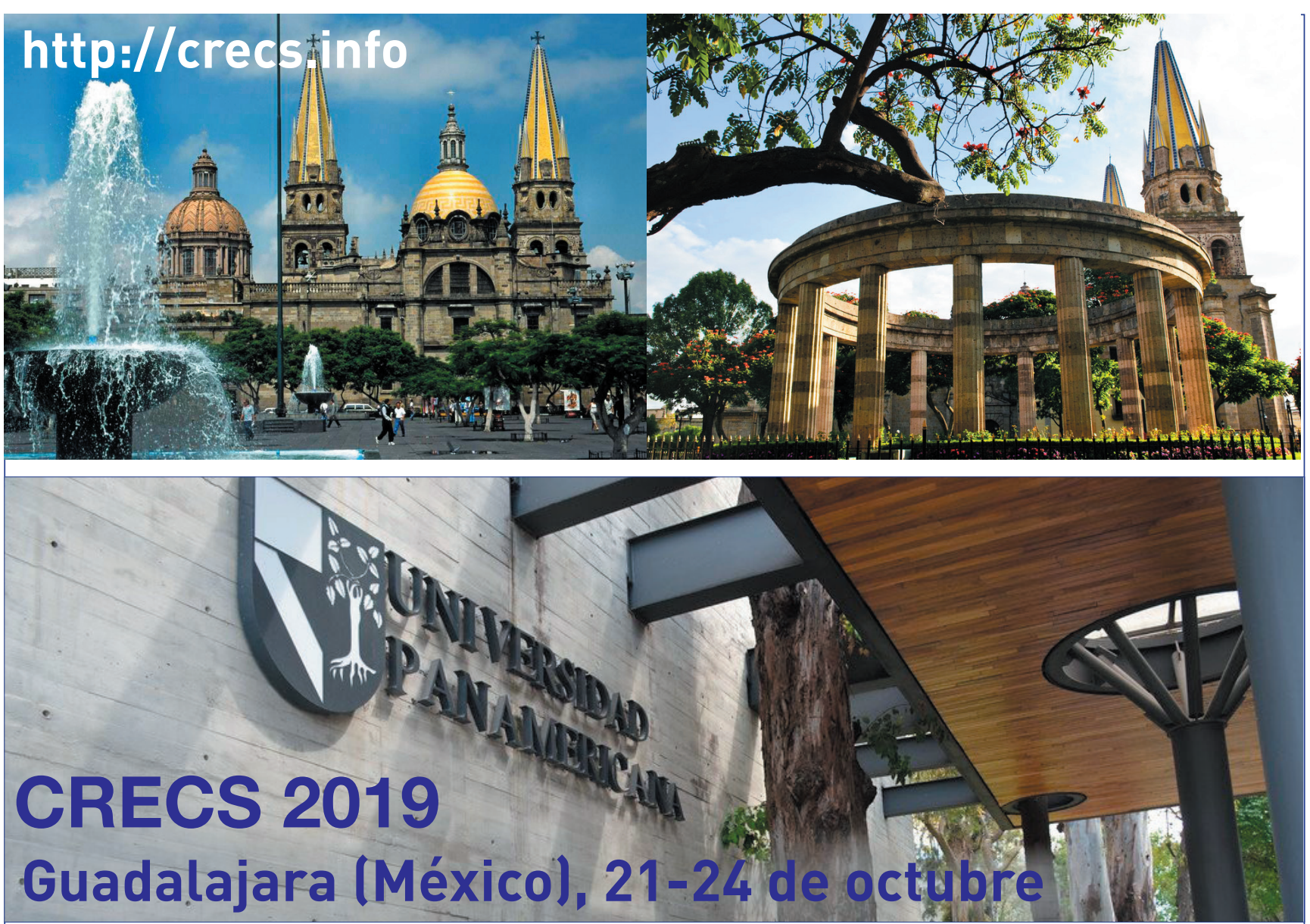

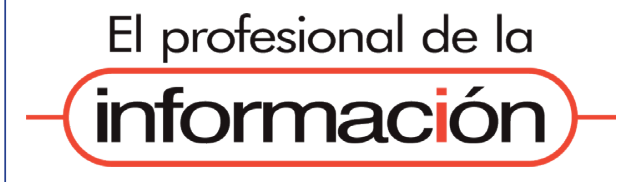

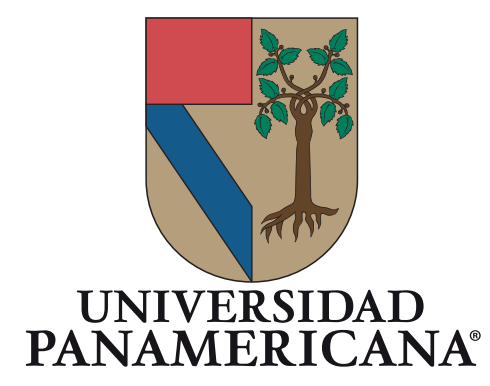

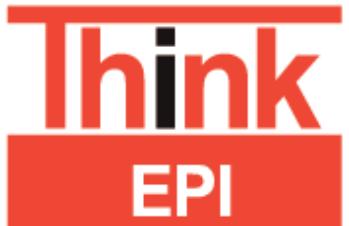

